Editorial

\title{
Statins and cancer mortality; do we need randomized, controlled trials?
}

\begin{abstract}
Much preclinical and epidemiologic evidence supports anticancer effects of HMG-CoA reductase inhibitors (Statins). Hyperlipidaemia is a well -established cardiovascular risk factor but the effect of hyperlipidaemia and treatment with cholesterol-lowering drugs on cancer remain equivocal. A reduction in the availability of cholesterol may limit the cellular proliferation required for cancer growth and metastasis.

Epidemiologic evidence supports a protective effect of statins on cancer prognosis but it does not support an association between statin use and reduced cancer incidence, but does. Supporting evidence comes from many investigators.

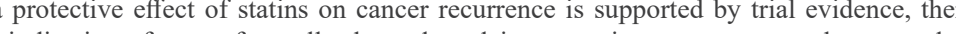
the indications for a safe, well-tolerated, and in expensive treatment can be expanded to improve outcomes for cancer survivors. We believe that the current evidence base is sufficient to justify a clinical trial of cancer adjuvant therapy with statins. We advocate for such a trial to be initiated without delay.
\end{abstract}

\author{
Volume 8 Issue I - 2017 \\ Hisham Atef \\ Clinical Oncology and Nuclear Medicine Department, Egypt \\ Correspondence: Hisham Atef, MD Chairman, Clinical \\ Oncology and Nuclear Medicine Department, Kasr AI Aini \\ Faculty of Medicine, Cairo University, Egypt, Tel (+20) I22 7| I \\ 2332, Email Hishamate@hotmail.com
}

Received: April 27, 2017 | Published: May 05, 2017

\section{Introduction}

Many in vitro and animal studies suggest that statin drugs suppress cancer progression. In human subjects, consistent evidence supports a protective effect on disease recurrence, but little evidence supports a protective effect of statins on breast cancer incidence.

The case for adjuvant breast cancer therapy with statins is unlikely to be advanced by new observational epidemiology studies, so evidence from a randomised trial is required. Our purposes herein are to provide a review of statin pharmacology, summarize the evidence for statin effects on cancer recurrence, and describe why a clinical trial is the next necessary step.

\section{Statin pharmacology}

Statins were discovered in 1976 with the isolation of mevastatin from Penicillium fungus.1 Several analogues were later marketed, all of which lower LDL cholesterol (LDL-C) concentrations in blood by inhibiting hydroxymethylglutaryl-coenzyme A reductase (HMGCoAR). HMGCoAR catalyses the conversion of 3-hydroxy-3methylglutaryl-coenzyme A (HMG-CoA) to mevalonic acid, a step in the mevalonate pathway that ultimately yields cholesterol. ${ }^{1}$

Statins bind to the active site of HMGCoAR with approximately 1,000 -fold greater affinity than HMG-CoA itself, resulting in strong competitive inhibition of the enzyme and arrested production of downstream compounds. ${ }^{2}$

\section{Supporting evidence}

Supporting evidence comes from many investigators including the work of Paul Carter et al, presented at the British CardioVascular Society, 2016.

They aimed to investigate the impact of comorbid diagnosis of hyperlipidaemia amongst patients with the four most prevalent cancer types in the United Kingdom (Lung, Breast, Prostate and Bowel). They did this using a large database of patients admitted with comorbid hyperlipidaemia to hospitals in the North of England, UK between 2000-2013.
Information on patients with a primary diagnosis of lung, breast, prostate and bowel cancers were obtained from hospitals in North England, UK between 1st January 2000 and 31stMarch 2013. This data was analysed according to the ACALM (Algorithm for Comorbidities, Associations, and length of stay and Mortality) study protocol.

Almost one million (929552) patients were admitted during the study period. Of these 7997 had lung cancer, 5481 had breast cancer, 4629 had prostate cancer, and 4570 had bowel cancer. Comorbid diagnoses of hyperlipidaemia significantly reduced mortality amongst patients with all four cancer types studied. Cox regression analyses accounting for differences in age, gender and ethnicity showed that hyperlipidaemia was associated with a significantly reduced mortality rate in lung cancer (OR $0.78,95 \%$ CI $0.70-0.87$ ), breast cancer (OR $0.57,95 \%$ CI $0.43-0.77$ ), prostate cancer (OR 0.53 , 95\% CI 0.50 0.79 ) and bowel cancer (OR $0.70,95 \%$ CI $0.58-0.84$ ).

They have concluded that comorbid hyperlipidaemia has a highly protective effect on mortality amongst patients with the four most prevalent cancers in the UK. The underlying reasons for this are yet to be determined but treatment with statins may contribute. This potentially beneficial effect of lipid-lowering medications amongst cancer patients should be further investigated. ${ }^{3}$

Statins impact on a significant reduction in cancer mortality was also concluding by two separate studies, one in women, and the other in men. Both were presented here at the American Society of Clinical Oncology (ASCO) 2015 Annual Meeting.

Specifically, statin use was associated with a $22 \%$ reduction in deaths from various cancer types in women and a $55 \%$ reduction in deaths from bone/connective tissue cancers. The study in men looked at statin use together with the antidiabetes medication metformin and found a $40 \%$ reduction in prostate cancer mortality, with the effect more pronounced in men with obesity/metabolic syndrome.

As for how such an effect is achieved, the researchers speculate that statins interfere with cell growth and metastasis by blocking cholesterol production, thereby affecting molecular pathways and the 
inflammatory response. The results in women were presented by Ange Wang.

Wang and colleagues examined data from the Women's Health Initiative (WHI), a 15-year research program involving postmenopausal women aged 50 to 79 years who were enrolled between 1993 and 1998 at 40 centers among 146,326 women. The median follow-up period was 14.6 years in the United States.

The researchers took into account a number of potential confounding factors, including age, race/ethnicity, education, smoking, body mass index, physical activity, family history of cancer, and current healthcare provider.

Multivariate analysis demonstrated that, compared with never having used statins, current statin use was associated with a significant reduction in cancer mortality, with an adjusted hazard ratio (aHR) of 0.78 vs never use $(\mathrm{P}<.0001)$. The association was unaffected by statin potency, lipophilicity/hydrophilicity, type, or duration. However statin use was not associated with cancer incidence, statin use was associated with significant reductions in deaths from breast $(\mathrm{aHR}=$ $0.60)$, ovarian $(\mathrm{aHR}=0.58)$, colorectal $(\mathrm{aHR}=0.57)$, digestive $(\mathrm{aHR}$ $=0.68)$, and bone/connective tissue cancers $(\mathrm{aHR}=0.45)$, but not from lung cancer $(\mathrm{aHR}=1.17){ }^{4}$

Although the validity of the results is supported by the prospective nature of the study, the criticism for that study emerges from the fact that, the post hoc analysis means that causality cannot be established. In fact "We don't know for sure if the link shown between a decreased risk of dying from cancer and statin use is due to the drugs themselves or some other reason". The other study, showing a reduction in prostate cancer mortality, was presented by Grace L. Lu-Yao, PhD. Her team looked at use of both statins and metformin.

The researchers used Surveillance, Epidemiology and End ResultsMedicare linked data to follow 22,110 patients diagnosed with highrisk prostate cancer, defined as a prostate-specific antigen (PSA) score of $\geq 20$, a Gleason score of $8-10$, or stage III or IV cancer.

Cox regression analysis was used to compare prostate cancerspecific mortality (PCSM) and covariates such as age, race, marital status, use of ADT, PSA and Gleason score at diagnosis, and comorbidity score. The majority of metformin users were also prescribed statins. Patients who took both statins and metformin (n $=1315$ ) were more likely than other patients to have a comorbidity score of $\geq 2$ and to have obesity/metabolic syndrome.

Patients who took metformin alone $(n=455)$ experienced no reduction in overall mortality or PCSM compared with those who used neither medication ( $\mathrm{n}=14,849 ; \mathrm{HR}, 1.04$ and 0.92 , respectively). However, patients who took both statins and metformin had a substantial reduction in both overall mortality and PCSM (HR, 0.66 and 0.57 , respectively). A similar pattern was seen in patients who took statins alone ( $n=4353$; HR, 0.75 and 0.60 , respectively).

The impact of combined statin and metformin therapy on overall mortality and PCSM was more pronounced in patients with documented obesity/metabolic syndrome, although the differences did not reach statistical significance.

Dr Lu-Yao concluded that, despite patients receiving combination therapy having more comorbidities and being more likely to have documented obesity/metabolic syndrome, statin and metformin therapy was associated with markedly better survival than patients taking metformin alone.
She suggested that, given that the majority of metformin users also take statins, the survival benefit observed in metformin users in the literature may be largely due to statin use. ${ }^{5}$

The criticism for that study is directed to the fact that the study was of relatively short duration and only $6.2 \%$ of the patients died of prostate cancer. The follow-up period was probably too short to show the impact of metformin, insofar as previous studies have shown that metformin needs to be taken for at least 3 years to have a survival benefit.

Evidence on the value of Statin use on survival for lung cancer comes from a population-based cohort study. Newly diagnosed lung cancer patients, from 1998 to 2009, identified from the English cancer registry data and linked to the UK Clinical Practice Research Datalink. Statistics mortality data up to the year 2012 were analyzed. In 3,638 lung cancer patients, there was evidence that statin use after diagnosis was associated with reduced lung cancer-specific mortality (adjusted HR, 0.89; 95\% CI, 0.78-1.02; $\mathrm{P}=0.09$ ). Associations were more marked after 12 prescriptions (adjusted HR, 0.81; 95\% CI, 0.67$0.98 ; \mathrm{P}=0.03$ ) and when lipophilic statins were investigated (adjusted HR, 0.81; 95\% CI, 0.70-0.94; $\mathrm{P}=0.01)$. Furthermore, in 11,051 lung cancer patients, statin use before diagnosis was associated with reduced lung cancer-specific mortality (adjusted HR, 0.88; 95\% CI, $0.83-0.93 ; \mathrm{P}<0.001)$. These findings provide support for conducting randomized controlled trials of statins as adjuvant cancer therapy in lung cancer patients. ${ }^{6}$

Further evidence comes from the Danish population who had received a diagnosis of cancer between 1995 and 2007, with follow-up until December 31, 2009. 18,721 had used statins regularly before the cancer diagnosis and 277,204 had never used statins. Multivariableadjusted hazard ratios for statin users, as compared with patients who had never used statins, were 0.85 (95\% confidence interval [CI], 0.83 to 0.87 ) for death from any cause and 0.85 (95\% CI, 0.82 to 0.87 ) for death from cancer. The reduced cancer-related mortality among statin users as compared with those who had never used statins was observed for each of 13 cancer types. ${ }^{7}$ On the other hand epidemiological findings have been inconsistent, as some have been limited by small sample sizes, as well as certain time-related biases.

A nationwide cohort study was conducted in Scotland aiming to investigate whether breast cancer patients who were exposed to statins had reduced breast cancer-specific mortality. In this retrospective cohort study of 15,140 newly diagnosed invasive breast cancer patients diagnosed from 2009 to 2012 within the Scottish Cancer Registry there was no evidence of an association between statin use and breast cancer-specific death (adjusted HR 0.93, $95 \%$ CI 0.77, 1.12). No significant associations were observed in dose-response analyses or in analysis of all-cause mortality. Overall, they found little evidence of a protective association between post-diagnostic statin use and cancer-specific mortality in a large nation-wide cohort of breast cancer patients. These findings will help the decision to conduct randomized controlled trials of statins as an adjuvant treatment in breast cancer ${ }^{8}$.

\section{Conclusion}

Hyperlipidaemia is a well -established cardiovascular risk factor but the effect of hyperlipidaemia and treatment with cholesterollowering drugs on cancer remains equivocal. A reduction in the availability of cholesterol may limit the cellular proliferation required for cancer growth and metastasis. Much preclinical and epidemiologic evidence supports anticancer effects of HMG-CoA reductase inhibitors 
(Statins) but it does not support an association between statin use and reduced cancer incidence.

If a protective effect of statins on cancer recurrence is supported by trial evidence, then the indications for a safe, well-tolerated, and in expensive treatment can be expanded to improve outcomes for cancer survivors. We believe that the current evidence base is sufficient to justify a clinical trial of cancer adjuvant therapy with statins.

\section{Funding}

None.

\section{Acknowledgements}

None.

\section{Conflicts of interests}

The authors declare that there is no conflict of interest.

\section{References}

1. Endo A. The origin of the statins. Atherosclerosis Supplements. 2004;5(3):125-130.
2. Bersot T. Drug Therapy for Hypercholesterolemia and Dyslipidemia. In: Brunton LL, Chabner BA, Knollman BC (Eds.), Goodman \& Gilman's The Pharmacological Basis of Therapeutics. (12th edn), McGraw-Hill, USA. 2011.

3. Paul Carter, Mcgowan J, Uppal H, et al. Hyperlipidaemia Reduces Mortality In Breast, Prostate, Lung And Bowel Cancer. British J Sports Med 102(supp 6). 2016.

4. Wang A, Aragaki AK, Tang JY, et al. (Statin use and all-cancer survival: prospective results from the Women's Health Initiative. Br J Cancer. 2016;115(1):129-135.

5. Grace L Lu-Yao. Combination statin/metformin and prostate cancer specific mortality: A population-based study. American Society of Clinical Oncology (ASCO) Annual Meeting. 2015.

6. Cardwell CR, Menamin UM, Hughes CM, et al. Cancer Epidemiol Biomarkers Prev. 2015;24(5):833-841.

7. Sune F Nielsen, Nordestgaard BG, Bojesen SE. Statin Use and Reduced Cancer-Related Mortality. N Engl J Med. 2012;367: 1792-1802.

8. Mc Menamin ÚC, Murray LJ, Hughes CM, et al. Statin use and breast cancer survival: a nationwide cohort study in Scotland. BMC Cancer. 2016;16:600. 